\title{
Rifampicin and isoniazid prophylactic chemotherapy for tuberculosis
}

\author{
L P Ormerod
}

\begin{abstract}
Prophylaxis of tuberculosis in children with four month $(n=53)$ and three month regimens $(n=213)$ of rifampicin and isoniazid from 1987 to 1996 were tolerated without any toxicity. The reduction in the proportion of paediatric tuberculosis, which was seen after the introduction of chemoprophylaxis with longer regimens in 1981, was maintained with the shorter duration regimens. Altered immigration patterns and a fall in the proportion of infectious tuberculosis, as defined by sputum culture positivity, have been excluded as factors in the reduced paediatric proportion of tuberculosis. The data show such regimens have little toxicity and provide indirect evidence that three months chemoprophylaxis may be as effective as longer regimens.

(Arch Dis Child 1998;78:169-171)
\end{abstract}

Keywords: tuberculosis; chemoprophylaxis; rifampicin; isoniazid

In 1987, we reported our experience with rifampicin and isoniazid as prophylactic treatment of children with strongly positive tuberculin tests. ${ }^{1}$ The introduction of such treatment had brought about a marked reduction in the incidence of paediatric tuberculosis in this high incidence district. ${ }^{2}$ A reduction in duration of treatment to six months for the years 1984-6 maintained the reduced incidence. ${ }^{1}$ Treatment duration with the two drugs was further reduced to four months during the years 1987-8 and to three months for the years 1989-96 inclusive. Other factors which could influence the pattern of tuberculosis, such as a decline in the proportion of infectious cases or the amount of new immigration, were also considered. Local screening of new immigrants has been done since 1982, with annual numbers of long stay immigrants at between 225 and 410 per year during $1983-8 .^{3}$ The numbers have remained greater than 400 per year from 1989 onwards, so a decline in new immigration can be excluded as a factor. Measures of deprivation (Jarman index) from 1981 and 1991 census data showed a slight increase in deprivation between those dates, so improved social conditions do not explain the changes.

Blackburn Infirmary, Blackburn, Lancs BB2 3LR

Correspondence to: Dr Ormerod.

Accepted 30 September 1997 a history of BCG vaccination; tuberculin conversion was documented; or they had a healed lesion in a chest radiograph. ${ }^{4-6}$ After initial chest radiography, isoniazid $10 \mathrm{mg} / \mathrm{kg}$ and rifampicin $10 \mathrm{mg} / \mathrm{kg}$ were prescribed. Liver function tests were not routinely done before or during treatment. Compliance was monitored by monthly visits from the tuberculosis nursing team who also checked with the pharmacists and general practitioners that regular prescriptions were being obtained. Patients had a repeat chest radiograph at three or four months, and were then discharged. The management was by the same staff as in the previous report using the same criteria. ${ }^{1}$

Since 1981, all notifications for tuberculosis in the district have been made from the chest clinic, which also receives all positive mycobacterial cultures and copies of histology suggesting tuberculosis from the district. Data on the bacteriological status of all notified cases of tuberculosis in 1981-96 were available and were analysed using positive sputum cultures for Mycobacterium tuberculosis as an index of infectious cases. The tuberculosis nursing service received details of all new immigrants in the district from the port of arrival system and through local links with the family health service authority continuously from $1982 .^{3}$ Only respiratory physicians treat adult tuberculosis (all forms) and paediatricians treat children with clinical tuberculosis. There were no changes in consultant staff in either department between 1981-94; two additional paediatricians were appointed in 1995. Analysis was by $\chi^{2}$ with continuity adjustment and MantelHaenszel test for linear association.

\section{Results}

Table 1 gives details of chemotherapy regimens and numbers treated. Table 2 shows the total notifications and those for children, by ethnic group, for 1981-96 inclusive. Three children notified with tuberculosis had been given chemoprophylaxis, one each in 1984, 1985, and 1986, out of 339 people given chemoprophylaxis between 1981-6 (0.6\%). No child notified in 1987-96 had received chemoprophylaxis before. The analysis shows that the proportion of children from the Indian subcontinent (ISC)

Table 1 Details of duration of chemoprophylaxis

\begin{tabular}{lll}
\hline Years of study & $\begin{array}{l}\text { Duration of treatment } \\
\text { regimen (months) }\end{array}$ & $\begin{array}{l}\text { No of } \\
\text { patients }\end{array}$ \\
\hline Nov 1981-Dec 1983 & 9 & $220^{\star}$ \\
$1984-6$ & 6 & $119\left(43^{\star}\right)$ \\
$1987-8$ & 4 & 53 \\
$1989-92$ & 3 & 134 \\
$1993-6$ & 3 & 79 \\
\hline
\end{tabular}

* Followed up for 15 months after cessation of chemoprophylaxis. 
Table 2 Notifications of children with tuberculosis 1981-96

\begin{tabular}{|c|c|c|c|c|c|}
\hline & \multicolumn{5}{|l|}{ Years } \\
\hline & $1981-3$ & $1984-6$ & $1987-8$ & 1989-92 & $1993-96$ \\
\hline Children from the ISC notified & 64 & 12 & 4 & 11 & 7 \\
\hline Total number of people from the ISC notified & 257 & 128 & 85 & 177 & 163 \\
\hline Children as $\%$ of total & 24.9 & 9.4 & 4.7 & 6.2 & 4.3 \\
\hline \multicolumn{6}{|l|}{$\chi^{2}$ with continuity correction } \\
\hline $1981-3$ & - & & & & \\
\hline $1984-6$ & $12.04^{\star \star}$ & - & & & \\
\hline $1987-8$ & $15.11^{\star \star}$ & 1.00 & - & & \\
\hline 1989-92 & $24.32^{\star \star}$ & 0.66 & 0.04 & - & \\
\hline $1993-6$ & $28.71^{\star \star}$ & 2.26 & 0.00 & 0.30 & - \\
\hline Mantel-Haenszel test of linear association & & & & $46.16^{\star \star}$ & \\
\hline White children notified & 27 & 7 & 0 & 1 & 1 \\
\hline Total white notifications & 129 & 88 & 38 & 57 & 34 \\
\hline Children as $\%$ of total & 20.9 & 8.0 & 0 & 1.75 & 2.9 \\
\hline \multicolumn{6}{|l|}{$\chi^{2}$ with continuity correction } \\
\hline $1981-3$ & - & & & & \\
\hline $1984-6$ & $5.72^{\star}$ & - & & & \\
\hline $1987-8$ & $8.01^{\star \star}$ & 1.86 & - & & \\
\hline 1989-92 & $9.92^{\star \star}$ & 1.50 & 0.00 & - & \\
\hline $1993-6$ & $4.92^{\star}$ & 0.35 & 0.00 & 0.00 & - \\
\hline \multicolumn{4}{|l|}{ Mantel-Haenszel test of linear association } & $19.99^{\star \star}$ & \\
\hline
\end{tabular}

Significance: ${ }^{\star} \mathrm{p}<0.05 ;{ }^{\star \star} \mathrm{p}<0.01$.

Table 3 Positive sputum cultures as a percentage of notifications

\begin{tabular}{|c|c|c|c|c|c|}
\hline & \multicolumn{5}{|l|}{ Years } \\
\hline & $1981-3$ & 1984-6 & $1987-8$ & 1989-92 & $1993-96$ \\
\hline Total ISC notifications & 237 & 128 & 85 & 179 & 163 \\
\hline Positive sputum cultures & 32 & 23 & 11 & 41 & 39 \\
\hline As $\%$ of total & 13.5 & 18.0 & 12.9 & 22.9 & 23.9 \\
\hline \multicolumn{6}{|l|}{$\chi^{2}$ with continuity correction } \\
\hline $1981-3$ & - & & & & \\
\hline $1984-6$ & 0.97 & - & & & \\
\hline $1987-8$ & 0.00 & 0.62 & - & & \\
\hline 1989-92 & $5.60^{\star}$ & 0.82 & 3.01 & - & \\
\hline $1993-6$ & $6.49^{\star}$ & 1.18 & 3.53 & 0.01 & - \\
\hline Mantel-Haenszel test of linear association & & & & $8.72^{\star \star}$ & \\
\hline Total white notifications & 129 & 88 & 38 & 57 & 34 \\
\hline Positive sputum cultures & 49 & 34 & 18 & 29 & 17 \\
\hline As $\%$ of total & 38 & 38.6 & 47.4 & 50.9 & 50 \\
\hline \multicolumn{6}{|l|}{$\chi^{2}$ with continuity correction } \\
\hline $1981-3$ & - & & & & \\
\hline $1984-6$ & 0.00 & - & & & \\
\hline $1987-8$ & 0.72 & 0.51 & - & & \\
\hline $1989-92$ & 2.20 & 1.64 & 0.02 & - & \\
\hline $1993-6$ & 1.15 & 0.88 & 0.00 & 0.00 & - \\
\hline Mantel-Haenszel test of linear association & & & & $3.86^{\star}$ & \\
\hline
\end{tabular}

Significance: ${ }^{\star} \mathrm{p}<0.05 ;{ }^{\star \star} \mathrm{p}<0.01$

and white children notified in 1981-3 was significantly greater than in later years, but that there were no differences in proportions after 1984-6. Table 3 shows the percentage of notified cases of tuberculosis with positive sputum cultures, as a measure of infectivity of those cases. The proportion of white infectious cases increased slightly over time although there was a fall in the absolute numbers. There was also an increase in the proportion of ISC notifications with positive cultures from 1989 onwards, with significant differences between 1989-92 and 1993-6 compared with 1981-3. No child required their treatment to be stopped for possible side effects between 1987-96 inclusive. Four children had their treatment modified because of nausea between 1981-6, but all had normal liver function.

\section{Discussion}

Prophylactic chemotherapy with rifampicin and isoniazid produced a greatly reduced proportion of paediatric notifications of tuberculosis within a few years of its introduction. ${ }^{1}$ The duration of treatment was then reduced to six months without loss of effect. ${ }^{1}$ Further reduction in the duration of chemoprophylaxis to four months for $1987-8$ and to three months for 1989-96 has shown no increase in the proportion of paediatric notifications in those years.

The significant reduction in the proportion of paediatric notifications in the ISC and white ethnic groups has been maintained. Although the absolute numbers of cases of tuberculosis have fallen among white people, the proportion of sputum positive cases has increased slightly. Only the use of chemoprophylaxis where appropriate seems to explain the significant reduction in the proportion of paediatric notifications in the white ethnic group as immigration was not a factor and there was no decline in the infectivity of cases. When considering the ISC notifications, no alteration or reduction, either between $1983-8^{3}$ or later, in the immigration pattern has been shown. The only change in infectivity of cases is an increase in the potentially infectious proportion from 1989 onwards, which if causing an interaction, should increase the proportion of paediatric cases, rather than the sustained fall that was seen. 
The evidence from this single health district over 15 years supports the view that the introduction of chemprophylaxis for strongly tuberculin positive children has significantly reduced the proportion of children with clinical tuberculosis. This reduction has been maintained as shorter durations of prophylaxis have been given, with three months of rifampicin and isoniazid being used for the past eight years. There have been no controlled trials of rifampicin and isoniazid prophylaxis in children. A controlled study in adults with silicosis, who have a substantially higher risk of developing tuberculosis, however, has shown that taking rifampicin and isoniazid for three months was as effective as taking isoniazid only for six months. ${ }^{7}$

The current study, although not a controlled trial, is an observational study over a prolonged period and was able to examine the possible effects of confounding factors. The data provide some indirect evidence to support the Joint Tuberculosis Committee's guidelines that such three month regimens may provide effective chemoprophylaxis for tuberculosis. ${ }^{8}$ No significant toxicity was observed in 266 patients treated with three or four month regimens. Children have a very low rate of drug reactions during treatment for clinical disease. ${ }^{9}$
These data suggest that little toxicity occurs from such chemoprophylaxis and that before and during treatment, liver function monitoring, in the absence of symptoms, is unnecessary.

1 Ormerod LP. Reduced incidence of tuberculosis by prophylactic chemotherapy in subjects showing strong reactions to tuberculin testing. Arch Dis Child 1987;82:1005-8.

2 Medical Research Council Tuberculosis and Chest Diseases Unit. Geographical distribution of tuberculosis notificaUnit. Geographical distribution of tuberculosis notifica-
tions in a national survey of England and Wales in 1983. tions in a national survey
Tubercle $1986 ; 86: 163-78$.

3 Ormerod L.P. Tuberculosis screening and prevention in new immigrants 1983-88. Respir Med 1990;84:269-71.

4 Joint Tuberculosis Committee of the British Thoracic Society. Control and prevention of tuberculosis: a code of practice. $B M \mathcal{F} 1983 ; 287: 1118-21$.

5 Joint Tuberculosis Committee of the British Thoracic Society. Control and prevention of tuberculosis in Britain: an ety. Control and prevention of tuberculosis in B
updated code of practice. BMF 1990;300:995-9.

6 Joint Tuberculosis Committee of the British Thoracic Society. Control and prevention of tuberculosis in the United Kingdom: code of practice 1994. Thorax 1994;49:11931200

7 Hong Kong Chest Service/Tuberculosis Research Centre Madras/British Medical Research Council. A double-blind placebo-controlled clinical trial of three anti-tuberculosis chemoprophylaxis regimens in patients with silicosis in Hong Kong. Am Rev Respir Dis 1992;145:36-41.

8 Joint Tuberculosis Committee of the British Thoracic Society. Chemotherapy and management of tuberculosis in the United Kingdom: recommendations of the Joint Tuberculosis Committee of the British Thoracic Society. Thorax 1990;45:403-8.

9 Ormerod LP, Horsfield N. Frequency and type of reactions to anti-tuberculosis drugs: observations in routine treatment. Tubercle and Lung Disease 1996:77:37-42. 